\title{
Static and Dynamic Statistical Correlations in Water: Comparison of Classical Ab Initio Molecular Dynamics at Elevated Temperature With Path Integral Simulations at Ambient Temperature
}

\author{
Chenghan Li, ${ }^{l}$ Francesco Paesani, ${ }^{2, *}$ and Gregory A. Voth ${ }^{l, *}$
}

'Department of Chemistry, Chicago Center for Theoretical Chemistry, James Franck Institute, and Institute for Biophysical Dynamics, The University of Chicago, Chicago, IL, 60637

${ }^{2}$ Department of Chemistry and Biochemistry, Materials Science and Engineering, and San Diego Supercomputer Center, University of California San Diego, La Jolla, California 92093

ABSTRACT. It is a common practice in ab initio molecular dynamics (AIMD) simulations of water to use an elevated temperature to overcome the over-structuring and slow diffusion predicted by most current density functional theory (DFT) models. The simulation results obtained in this distinct thermodynamic state are then compared with experimental data at ambient temperature based on the rationale that a higher temperature effectively recovers nuclear quantum effects (NQEs) that are missing in the classical AIMD simulations. In this work, we systematically examine the foundation of this assumption for several DFT models as well as for the many-body MB-pol model. We find for the cases studied that a higher temperature does not correctly mimic NQEs at room temperature, which is especially manifest in significantly different three-molecule correlations as well as hydrogen bond dynamics. In many of these cases, the effects of NQEs are the opposite of the effects of carrying out the simulations at an elevated temperature. 
KEYWORDS. ab initio molecular dynamics, water structure and dynamics, nuclear quantum effects

\section{INTRODUCTION}

Due to the light mass of hydrogen nuclei, nuclear quantum effects (NQEs) are sometimes assumed to be important for a quantitative modeling of the structural, thermodynamic, and dynamical properties of liquid water. ${ }^{1}$ Though NQEs appear to be minimal for the calculated intermolecular properties in some water simulations, ${ }^{2-3}$ they were found to be non-negligible in other $\operatorname{cases}^{4-5}$ as demonstrated by the differences in thermodynamic properties between light and heavy water. ${ }^{1}$

In principle, Feynman's imaginary-time path-integral formalism ${ }^{6}$ enables the modeling of NQEs in liquid water to numerical accuracy, but the associated high computational cost has hindered widespread application of path-integral molecular dynamics (PIMD) simulations until recent developments of more efficient approximations, ${ }^{7}$ such as the ring-polymer contraction (RPC), ${ }^{8}$ the ring-polymer interpolation, ${ }^{9}$ and the combined path-integral and generalized Langevin equation (PI+GLE) approach. ${ }^{10}$ The computational cost of a PIMD simulation of liquid water significantly increases when the underlying Born-Oppenheimer potential energy surface is calculated "on the fly" as in ab initio molecular dynamics (AIMD) simulations ${ }^{11}$ where Kohn-Sham density functional theory ${ }^{12}$ (KS-DFT) is generally used to solve the (electronic) Schrödinger equation at each step of the dynamical trajectory. As a consequence, most of the AIMD simulations reported in the literature have been conducted ignoring NQEs and treating the nuclei as classical particles.

Among existing exchange-correlation functionals, generalized-gradient approximation (GGA) functionals have been extensively used in AIMD simulations of liquid water due to their relatively lower computational cost. GGA functionals typically overestimate the strength of the hydrogen bonds in water. This results in over-structuring of the liquid phase which is accompanied by slow 
molecular diffusion and, in some cases, glassy-like behavior. ${ }^{13}$ The inclusion of dispersion corrections was found to partially alleviate these problems. ${ }^{13}$

An alternative and $a d$ hoc approach adopted in the literature to overcome over-structuring and slow diffusion in AIMD simulations of liquid water consists of performing the simulations at a higher temperature. While simulations at a higher temperature sample a different thermodynamic state, it is sometimes implicitly assumed that the extra thermal energy effectively mimics NQEs that are missing from the classical AIMD simulations at room temperature. Within this assumption, the results obtained from classical AIMD simulations carried out at higher temperature are thus sometimes considered to be equivalent to the actual results at room temperature with NQEs included.

Although there has been discussion on the temperature dependence of NQEs for empirical and data-driven water models,,$^{14-17}$ and the differences between classical and quantum radial distribution functions (RDFs), diffusion coefficients, and single hydrogen-bond behavior at $300 \mathrm{~K}$ and $330 \mathrm{~K}$ have been noted, ${ }^{1,18}$ a clear justification for the use of elevated-temperature AIMD simulations to recover NQEs has not been systematically established or examined. It should be noted that one of these studies has also involved higher-level DFT descriptions in the AIMD, in a quest to find the most accurate AIMD model for liquid water. ${ }^{18}$ To our knowledge, none of these prior studies have provided an examination of static and dynamic statistical correlations involving more than two water molecules, which is a primary result of the present work.

Herein, we employ the RPC method to explicitly model NQEs at room temperature and systematically benchmark NQEs on structural correlations as well as dynamical properties of liquid water at room temperature against classical MD simulations carried out at higher temperature (and especially including multi-molecule correlations). Our analysis includes three 
exchange-correlation functionals: (1) the strongly constrained and appropriately normed (SCAN) functional which is a meta-GGA functional, ${ }^{19}(2)$ the popular Becke-Lee-Yang-Parr functional20-21 with D3 dispersion correction ${ }^{22}$ (BLYP-D3), and (3) the more recently developed BLYP-D3 functional with an experiment directed simulation (EDS) correction. ${ }^{23}$ (The EDS correction employs a minimal bias to improve the BLYP-D3 description of hydrogen bonding in liquid water, and has been shown to provide significantly more accurate water properties. ${ }^{23}$ ) Additionally, we perform the same analyses using MB-pol, ${ }^{24-26}$ arguably the most accurate water potential developed to date. MB-pol is a data-driven model rigorously derived from the many-body expansion of the interaction energies calculated at the coupled cluster level of theory - a model which has been shown to accurately predict the properties of water in various forms, from small gas-phase clusters to liquid water and ice. ${ }^{27-28}$ We note that the goal of our present study is not to determine which water model and simulation protocol best reproduces the experimental data, but to assess the validity of using an elevated temperature in classical MD simulations of liquid water to effectively model NQEs at ambient temperature.

\section{METHODS}

The BLYP-D3 and EDS-BLYP-D3 simulations were performed with 128 water molecules in a cubic simulation box of side $L=15.64 \AA$. The Goedecker-Teter-Hutter $(\mathrm{GTH})$ pseudopotentials ${ }^{29}$ were used to model the core electrons, while a TZV2P basis set was used to expand the KohnSham orbitals and a plane wave basis set with a cutoff of 400 Ry was used to expand the electron density. The orbital transformation (OT) method $^{30}$ was used to optimize the wave function at each step, using a self-consistent field (SCF) convergence criterion of $1 \times 10^{-7}$ a.u. The SCAN simulations were performed with 64 water molecules in a cubic box of side $\mathrm{L}=12.66 \AA$ to be 
consistent with the setup in $\operatorname{ref}^{31}$. Similar to the BLYP-D3 simulations, the TZV2P basis set was used but a larger plane wave basis set with a cutoff of 600 Ry was used for better SCF convergence. The GTH pseudopotentials optimized for SCAN were used to model the core electrons (https://github.com/juerghutter/GTH). The OT method was used to optimize the wave function and the SCF convergence criterion was the same as BLYP-D3.

For the classical AIMD simulations with BLYP-D3 and EDS-BLYP-D3, the system was equilibrated in the canonical (constant NVT) ensemble for 90 ps at $298 \mathrm{~K}$ and for 85 ps at $328 \mathrm{~K}$. In the case of SCAN, the NVT equilibration was carried out for 70 ps at $298 \mathrm{~K}$ and for 72.5 ps at 328 K. In all simulations, a Nosé-Hoover chain thermostat with a characteristic frequency of 3000 $\mathrm{cm}^{-1}$ was coupled to all degrees of freedom, and a timestep of $0.5 \mathrm{fs}$ was used to integrate Newton's equations of motion. All simulations were carried out with the CP2K software package. ${ }^{32-33}$ The EDS correction was added via a modified version of PLUMED2 ${ }^{34}$ coupled to CP2K, with the EDS parameters taken from ref $^{23}$, while the simulations with the SCAN exchange-correlation functional were carried out by linking the Libxc library ${ }^{35}$ to $\mathrm{CP} 2 \mathrm{~K}$.

The dynamical properties were calculated by performing MD simulations in the microcanonical (constant NVE) ensemble which were started from configurations previously equilibrated in the canonical ensemble. At each temperature, five independent replicas were launched from the NVT trajectories at intervals of $10 \mathrm{ps}$, and were carried out for 30-40 ps. The multi-time stepping (MTS) scheme was used to accelerate the simulations, with an inner timestep of $0.25 \mathrm{fs}$ and an outer timestep of 2 fs. For the reference potential used in the MTS scheme, we used a deep learning potential (DP) trained on BLYP-D3, EDS-BLYP-D3, and SCAN data. The training set for BLYPD3 contained 2500 configurations sampled at $298 \mathrm{~K}$ and 2500 configurations sampled at $328 \mathrm{~K}$. The training set for EDS-BLYP-D3 contained 132000 configurations sampled at $298 \mathrm{~K}$. The 
training set for SCAN contained 10000 configurations sampled at $298 \mathrm{~K}$ and 10000 configurations sampled at $328 \mathrm{~K}$. Both energies and forces were used in the training. The training was conducted using the DeepMD-kit tool ${ }^{36}$ with the smooth edition of deep potential molecular dynamics (DPMD). ${ }^{37}$ The BLYP-D3 energies and forces were calculated with CP2K, the forces for the DP potential were computed using LAMMPS ${ }^{38}$ coupled to DeepMD-kit, the EDS correction was computed using PLUMED2, and the PIMD simulations were performed using the i-Pi force engine. ${ }^{39}$ The averaged structural properties obtained from NVE simulations were found to be identical with those obtained in the NVT ensemble. An independent NVE simulation was carried out with a timestep of $0.5 \mathrm{fs}$, without applying the MTS approximation, to calculate the diffusion coefficient which was found to be identical to the value obtained from an analogous simulation carried out using the MTS scheme.

Approximate quantum dynamics simulations were carried out using thermostatted ring-polymer molecular dynamics (TRPMD) ${ }^{40}$ Following ref $^{5}$, the RPC approach was employed with $P^{\prime}=1$, i.e., the centroid contraction. The path integral of each atom was discretized with $P=30$ beads. All the static property analyses were performed both on the centroid coordinates and on all the path integral beads, while the dynamical properties were computed from the centroid trajectories. The same reference potential used in MTS was used in RPC. Five independent TRPMD trajectories of $30-40$ ps each were performed to calculate the dynamical properties. Classical and quantum MB-pol trajectories were taken from refs ${ }^{26-27,41}$. 

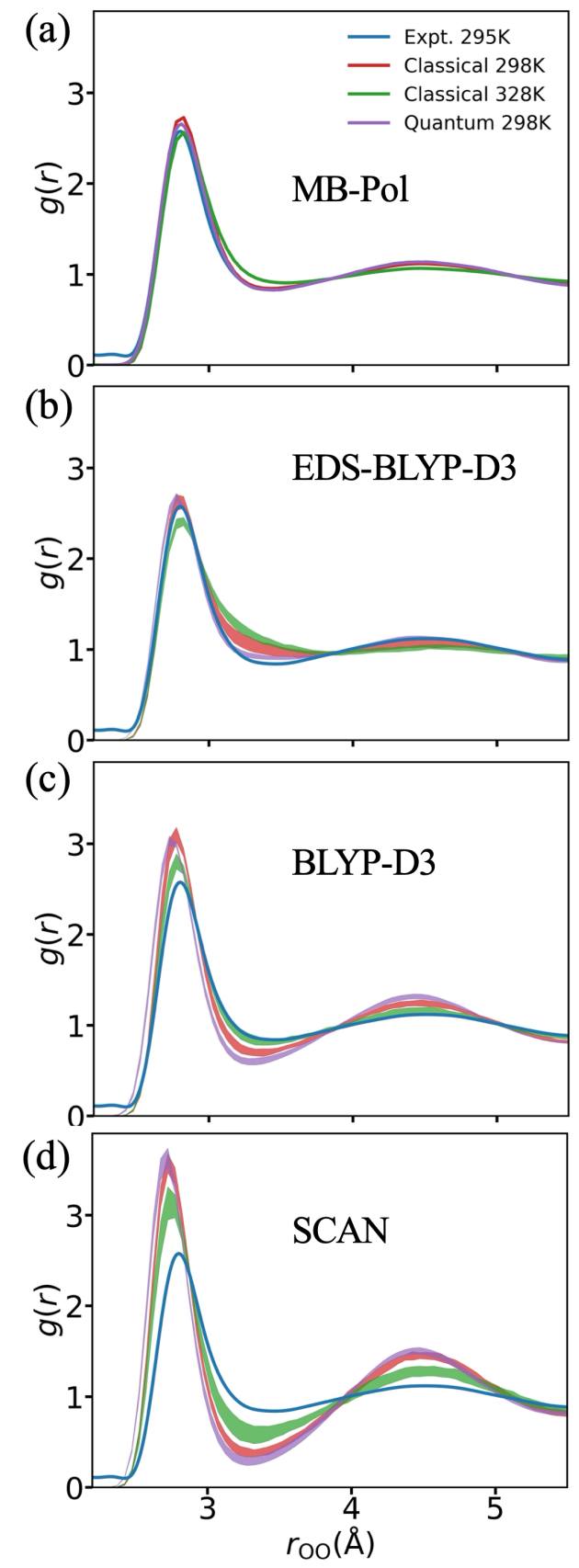

Figure 1. O-O RDFs of (a) MB-pol, (b) EDS-BLYP-D3, (c) BLYP-D3 and (d) SCAN water model at $298 \mathrm{~K}$ with classical nuclei (red) and with quantized nuclei (purple), and at $328 \mathrm{~K}$ (green). The experimental value ${ }^{42}$ at $295 \mathrm{~K}$ is plotted in blue. Statistical errors computed from independent runs are shown by the curve widths. The quantum results were computed as the averages over path integral beads, while the results computed from the particle imaginary time path centroid coordinates are presented in Figure S1 of the Supporting Information (SI). 

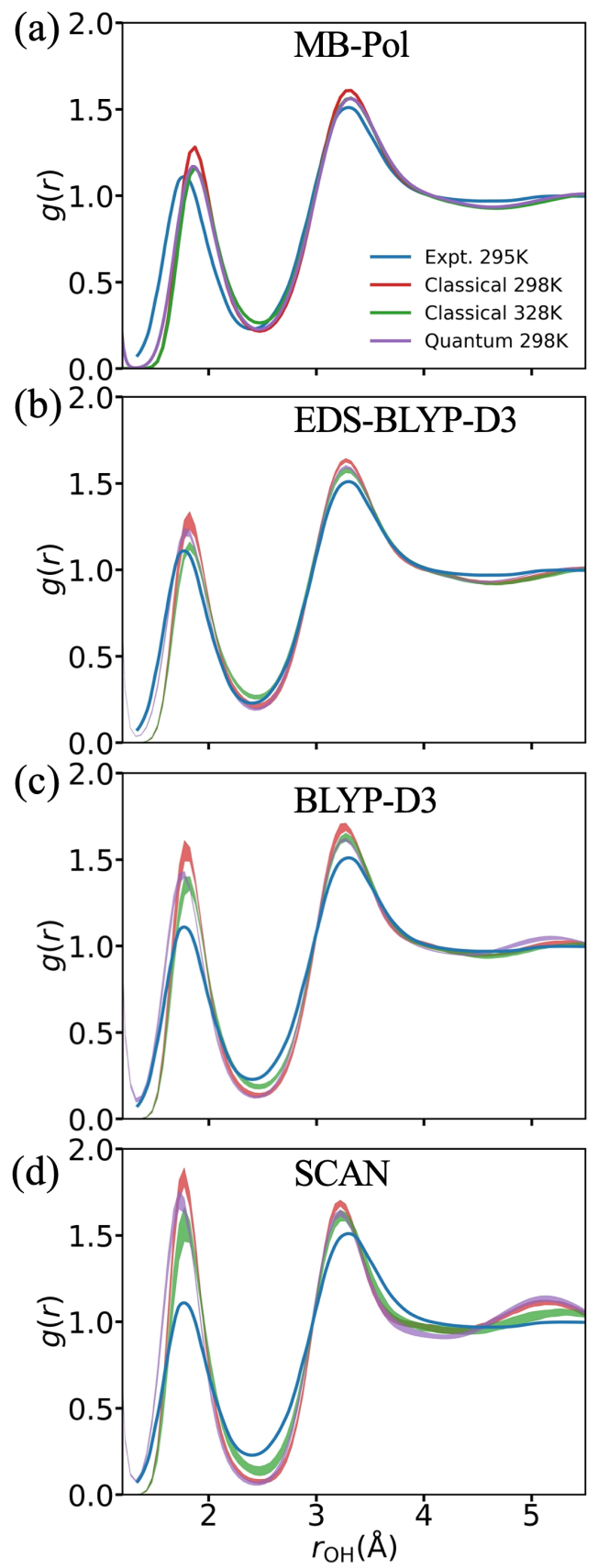

Figure 2. O-H RDFs of (a) MB-Pol, (b) EDS-BLYP-D3, (c) BLYP-D3 and (d) SCAN water model at $298 \mathrm{~K}$ with classical nuclei (red) and with quantized nuclei (purple), and at $328 \mathrm{~K}$ (green). The experimental value ${ }^{43}$ at $298 \mathrm{~K}$ is plotted in blue. Statistical errors computed from independent runs are shown by the curve widths. The quantum results were computed as the averages over path integral beads, while the results computed from the particle imaginary time path centroid coordinates are presented in Figure S2 of the SI. 

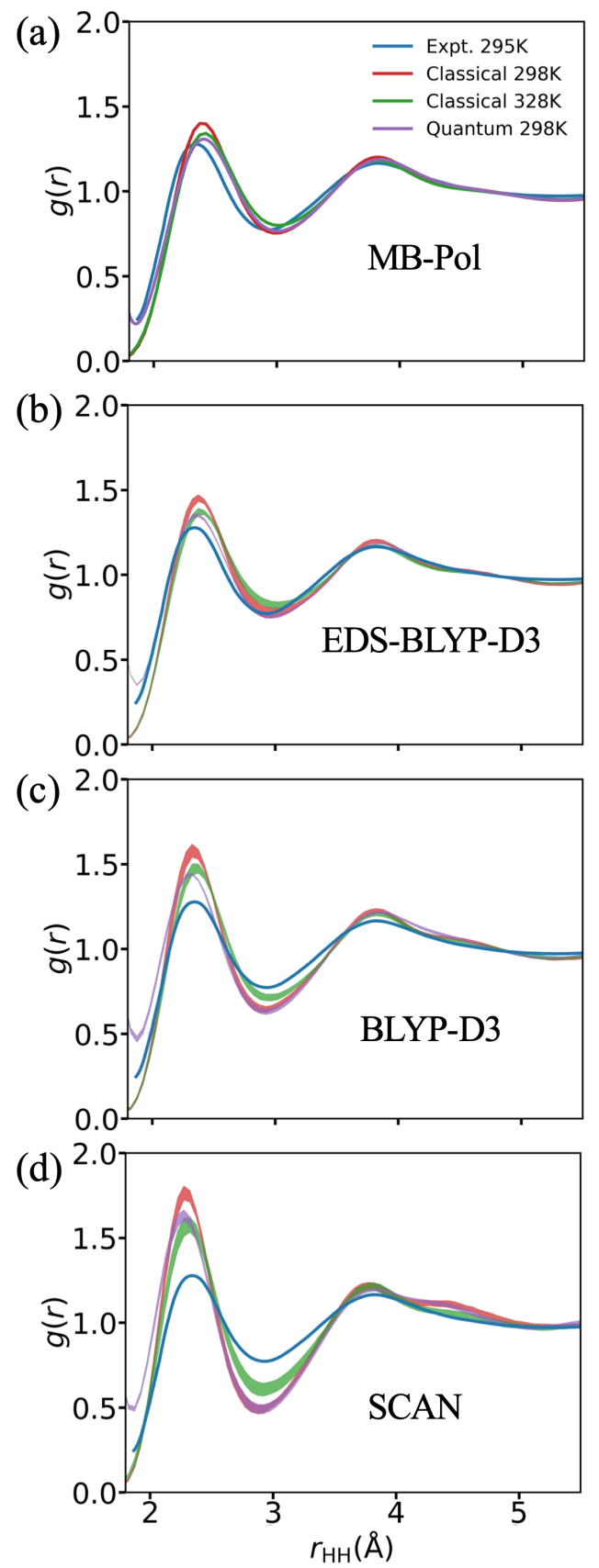

Figure 3. H-H RDFs of (a) MB-Pol, (b) EDS-BLYP-D3, (c) BLYP-D3 and (d) SCAN water model at $298 \mathrm{~K}$ with classical nuclei (red) and with quantized nuclei (purple), and at $328 \mathrm{~K}$ (green). The experimental value ${ }^{43}$ at $298 \mathrm{~K}$ is plotted in blue. Statistical errors computed from independent runs are shown by the curve widths. The quantum results were computed as the averages over path integral beads, while the results computed from the particle imaginary time path centroid coordinates are presented in Figure S3 of the SI. 


\section{RESULTS AND DISCUSSION}

We analyzed simulations from the four water models (BLYP-D3, EDS-BLYP-D3, SCAN, and MB-pol) at ambient temperature $(298 \mathrm{~K})$ and at an elevated temperature (328 K) commonly used to effectively mimic NQEs. The simulations at $298 \mathrm{~K}$ were performed with both classical and quantized nuclei, while classical nuclei were used at $328 \mathrm{~K}$.

Figure 1-3 show the radial distribution functions of the four water models. As seen in Figure 1(a), NQEs only have a minimal effect on the oxygen-oxygen two-body correlation in MB-pol water, with the first peak in the O-O RDF being slightly less structured with quantized nuclei, which, in turn, slightly improves the agreement with the experimental data. NQEs show an opposite effect in the three DFT models where all the O-O RDFs become more structured with quantized nuclei. Similar results were found for the B97M-rV and revPBE0-D3 density functionals in ref. ${ }^{18}$. Notably, when NQEs are explicitly accounted for, the hydrogen bonds in DFT water shrink as shown by the first peak in the O-H and H-H RDFs (Figure $2 \& 3(\mathrm{~b})-(\mathrm{d})$ ) moving towards shorter distances (purple vs. red curves). This agrees with the experimental observation of shorter hydrogen bond length in light water than heavy water. ${ }^{44}$ On the other hand, this shift is not observed in the quantum O-H and H-H RDFs of MB-pol. Despite these differences, NQEs are predicted to have similar effects on the $\mathrm{O}-\mathrm{H}$ and $\mathrm{H}-\mathrm{H}$ RDFs calculated with the four water models, resulting in overall less structured RDFs. The effect associated with an elevated temperature is more consistent among the four water models - the extra thermal energy reduces the solvation structure in all the RDFs. It follows that performing classical MD simulations at an elevated temperature apparently mimics NQEs in simulations with MB-pol, while it has often opposite effects in simulations with the three DFT models. In fact, NQEs make the O-O RDFs calculated with BLYP-D3 and SCAN even more structured, which is consistent with the more structured water seen in quantum AIMD 
simulations with several DFT functionals reported in refs ${ }^{5,18}$. It should be noted that including NQEs improves the agreement between the EDS-BLYP-D3 and experimental RDFs. The consequences of NQEs compared to a higher temperature simulation can also be seen in the centroid-centroid RDFs (Figures S1-S3 of the SI).

We next examine three-body (i.e., three water molecule) correlations by computing the tetrahedral order parameter $q$ defined as ${ }^{45}$

$$
q=1-\frac{3}{8} \sum_{i \neq j}\left(\cos \theta_{i j}+\frac{1}{3}\right)^{2},
$$

where $\theta_{i j}$ is the $\mathrm{O}_{i}-0-\mathrm{O}_{j}$ angle centered on a given oxygen $\mathrm{O}$, and the sums are over the four closest oxygen atoms around 0 . The value of $q$ provides a measure of tetrahedral order in the liquid, with a value of 1 corresponding to a perfect tetrahedral arrangement and a value of 0 representing the ideal gas limit. Figure 4 depicts the distribution of $q$ for the four water models. Similar to the O-O RDF, NQEs still play a small role in determining the three-body correlations in the MB-pol simulations at ambient temperature. However, more pronounced differences are found in the simulations with the three DFT-based AIMD models. These have the quantum peak at $q \approx 0.85$ increasing and the peak at $q \approx 0.5$ decreasing relative to the classical curves, which is consistent with the more structured RDFs observed in Figure 1. In contrast, increasing the temperature has the opposite effect of reducing the tetrahedral structure in all four water models, which makes the distributions obtained with BLYP-D3 and SCAN qualitatively more similar to the distribution calculated with MB-pol at ambient temperature. It should be noted that this apparent better agreement with the MB-pol distribution is the result of fortuitous error cancellation associated with intrinsic deficiencies in the ability of BLYP-D3 and SCAN to represent water and not a consequence of NQEs, since when NQEs are explicitly taken into account in the simulations, 
all DFT models predict a significantly more tetrahedral structure. This behavior for all DFT models is exactly the opposite of the effect on the three-body (three-molecule) correlations due to a higher temperature. The three-body analysis for the path centroid coordinates show similar results (Figure S4 of the SI).
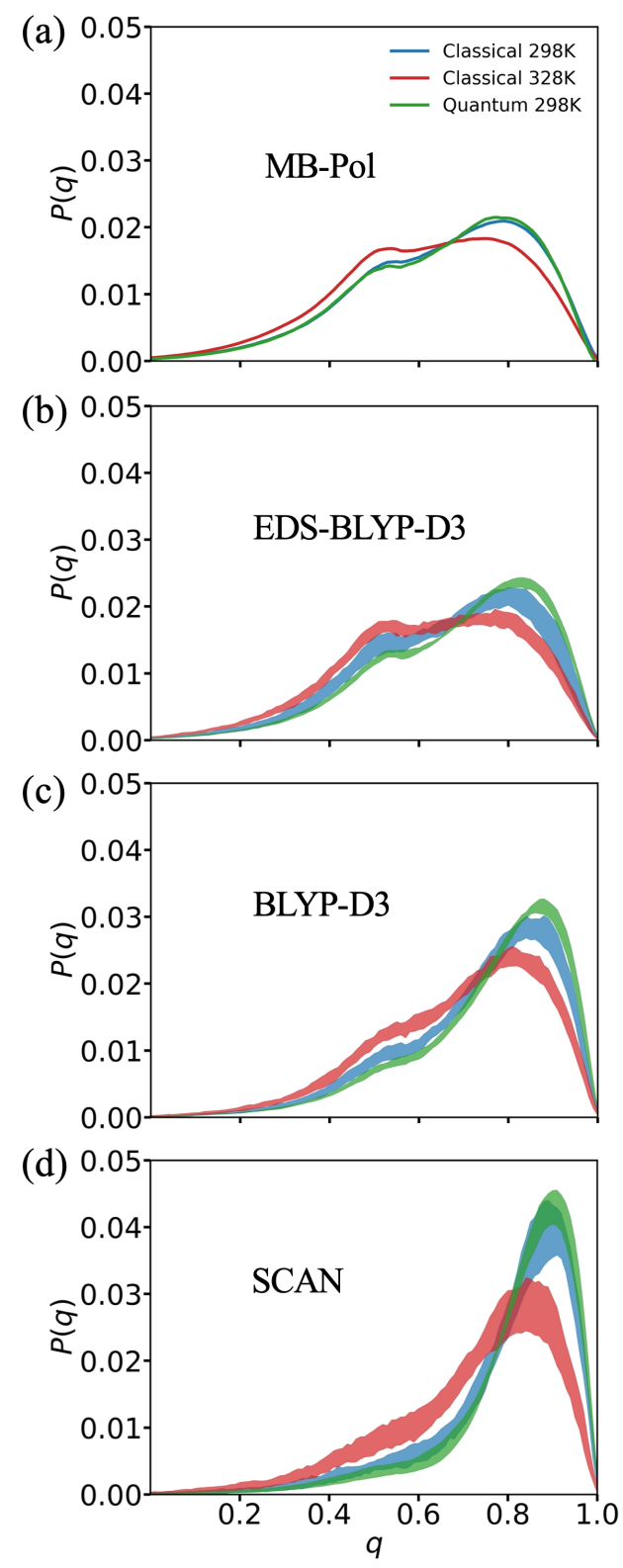

Figure 4. Tetrahedral order parameter $q$ distribution of (a) MB-Pol, (b) EDS-BLYP-D3, (c) BLYP-D3 and (d) SCAN water model at 298K with classical nuclei (blue) and with quantized nuclei (green), and at $328 \mathrm{~K}$ (red). Statistical errors computed from independent runs are shown by the curve widths. The quantum results were computed as the averages over path integral beads, while the results computed from the particle imaginary time path centroid coordinates are presented in Figure S4. 
To better determine how the additional thermal energy available at $328 \mathrm{~K}$ perturbs the system dynamics and depending on the underlying water model, we computed the hydrogen-bond dynamics and water self-diffusion constant at both temperatures, with and without including NQEs. In this analysis, we consider that molecule $j$ is hydrogen-bonded to molecule $i$ if the $\mathrm{O}_{i}-$ $\mathrm{O}_{j}$ distance is shorter than $3.5 \AA$ and the $\mathrm{H}_{i}-\mathrm{O}_{i}-\mathrm{O}_{j}$ angle is smaller than $30^{\circ}$, where $\mathrm{H}_{i}$ is one of the two bonded hydrogen atoms to $\mathrm{O}_{i}$. At a given time $t$, the hydrogen bond matrix is computed as

$$
h_{i j}=\left\{\begin{array}{ll}
1, & j \text { hydrogen }- \text { bonded to } i \\
0, & \text { otherwise }
\end{array},\right.
$$

The corresponding hydrogen-bond correlation function is defined as

$$
\langle h(0) h(\tau)\rangle=\frac{1}{N_{\text {wat }}\left(N_{\text {wat }}-1\right)} \sum_{i \neq j}\left\langle h_{i j}(0) h_{i j}(\tau)\right\rangle .
$$

In the analysis of the quantum simulations with the DFT-based AIMD models, we also observed spurious water auto-ionization approximately $5 \%$ of the time. Due to the ambiguity in assigning bonded hydrogens to oxygens with auto-ionized water, the hydrogen bond matrix $h_{i j}$ is set to be the value at the closest time when the bonding topology is well defined, i.e., when water autoionization does not occur. This transient auto-ionization was also reported in ref $^{46}$ and is likely the consequence of NQEs further reducing the proton transfer barrier between two water molecule which is already underestimated in the DFT models of water. ${ }^{47}$ As such, NQEs strengthen the hydrogen bonds and make DFT water more structured as shown in Figure 1 and Figure 4, which then also results in a slower hydrogen-bond dynamics as shown in Figure 5. As expected, simulations carried out at $328 \mathrm{~K}$ display an accelerated hydrogen-bond dynamics as seen in the more rapid decays of the corresponding hydrogen-bond correlation functions, which is exactly the opposite of the effect of NQEs in these DFT models. In MB-pol, the NQEs have little effect on the 
h-bond dynamics, while the higher temperature speeds them up to a certain degree, as expected. It is to be noted that h-bond dynamics (time correlations) are a collective phenomenon in liquid water that involves multiple water molecules and not simply confined to breaking of a single $\mathrm{H}$...O-H h-bond. ${ }^{48}$
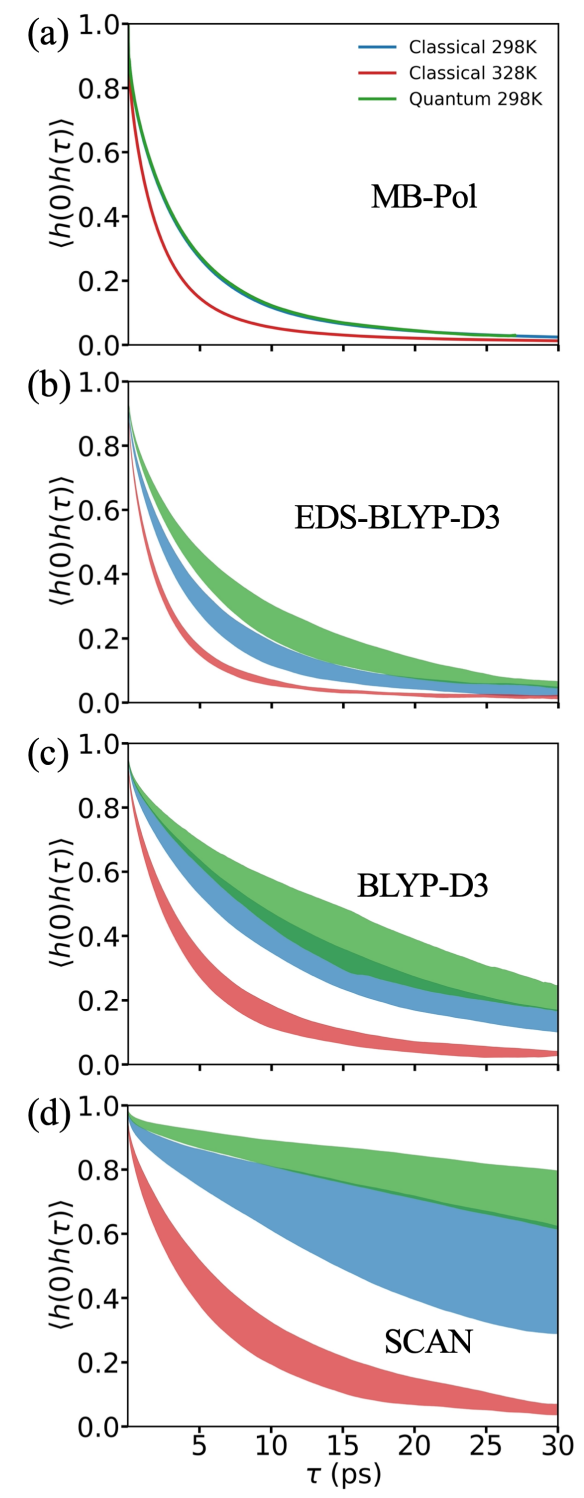

Figure 5. Hydrogen bond correlation function of (a) MB-Pol, (b) EDS-BLYP-D3, (c) BLYP-D3 and (d) SCAN water model at $298 \mathrm{~K}$ with classical nuclei (blue) and with quantized nuclei (green), and at $328 \mathrm{~K}$ (red). Statistical errors computed from independent runs are shown by the curve widths. 
We further investigated water dynamics by computing the self-diffusion constant from a linear fit to the 5 ps -15 ps segment of the mean-square displacement (MSD), defined as

$$
\operatorname{MSD}(t)=\left\langle\left(\boldsymbol{r}_{\mathrm{O}}(t)-\boldsymbol{r}_{\mathrm{O}}(0)\right)^{2}\right\rangle
$$

where $\boldsymbol{r}_{\mathrm{O}}$ represents the oxygen position of a water molecule. This function is typically averaged over all water molecules in the homogeneous liquid system. The computed values are summarized in

Table 1. We also report the diffusion constants after a correction for the finite simulation box used in simulations via ${ }^{49}$

$$
D(\infty)=D(L)+\frac{\xi k_{B} T}{6 \pi \eta L}
$$

where $\xi=2.837297$ is a constant for cubic boxes, $k_{B}$ is the Boltzmann constant, $T$ is the simulation temperature, and $L$ is the simulation box side length. In evaluating eq 5 , the experimentally determined viscosity of water $\eta$ was used,${ }^{50}$ which results in an overestimation of $D(\infty)$ for the BLYP-D3 and SCAN models since the over-structuring predicted by these two models would actually be associated with a viscosity higher than the experimental value (if the viscosity could be readily calculated from the AIMD, which it cannot). Clearly, for the three DFT models, the NQEs slows down the water self-diffusion while the elevated temperature, in a exactly clearly opposite trend, accelerates the diffusion dynamics. These results are also in line with those reported for the B97M-rV and revPBE0-D3 functionals in ref ${ }^{18}$. For MB-pol, in spite of a slower hydrogen-bond dynamics, NQEs do not introduce a significant difference in the diffusion constant at ambient temperature, while, as expected, a much faster diffusion is observed at $328 \mathrm{~K}$. 
Table 1. Self-diffusion constants of water in $\AA^{2} /$ ps.

\begin{tabular}{|c|c|c|c|c|c|}
\hline Model & & MB-Pol & $\begin{array}{c}\text { EDS-BLYP- } \\
\text { D3 }\end{array}$ & BLYP-D3 & SCAN \\
\hline \multirow{2}{*}{$\begin{array}{c}\text { Diffusion } \\
\text { Constant }\end{array}$} & Classical 298K & $0.23 \pm 0.01$ & $0.19 \pm 0.03$ & $0.08 \pm 0.02$ & $0.03 \pm 0.02$ \\
\cline { 2 - 6 } & Classical 328K & $0.38 \pm 0.05$ & $0.32 \pm 0.03$ & $0.20 \pm 0.03$ & $0.14 \pm 0.04$ \\
\cline { 2 - 6 } & Quantum 298K & $0.23 \pm 0.05$ & $0.13 \pm 0.02$ & $0.06 \pm 0.03$ & $0.009 \pm 0.005$ \\
\hline $\begin{array}{c}\text { Diffusion } \\
\text { Constant after } \\
\text { Size Correction }\end{array}$ & Classical 298K & $0.27 \pm 0.01$ & $0.23 \pm 0.03$ & $0.12 \pm 0.02$ & $0.09 \pm 0.02$ \\
\cline { 2 - 7 } & Classical 328K & $0.42 \pm 0.05$ & $0.37 \pm 0.03$ & $0.25 \pm 0.03$ & $0.20 \pm 0.04$ \\
\cline { 2 - 7 } & Quantum 298K & $0.27 \pm 0.05$ & $0.17 \pm 0.02$ & $0.10 \pm 0.03$ & $0.069 \pm 0.005$ \\
\hline Experimental ${ }^{51}$ & $298 \mathrm{~K}$ & \multicolumn{3}{|c|}{0.23} \\
\cline { 2 - 6 } & $329 \mathrm{~K}$ & \multicolumn{3}{|c|}{0.44} \\
\hline
\end{tabular}

Although it is not the main focus of this study to compare water models relative to the experimental data, it is worth noting that, among the four models considered in our analyses, MBpol provides the most accurate description of water at both temperatures, followed by the EDS corrected BLYP-D3 model. Both structural and dynamical properties of BLYP-D3 at $328 \mathrm{~K}$ are accidentally close to the experimental values determined at $298 \mathrm{~K}$ but not as the result of that system at a higher temperature effectively mimicking NQEs.

\section{CONCLUSIONS}

In this study, we systematically investigated the effect of an elevated temperature in simulating water with AIMD, and with a particular focus on a suggested empirical relationship between higher 
temperature and nuclear quantum effects at room temperature. We also were especially focused on the static and dynamic correlations between three or more water molecules. We considered three DFT-based AIMD water models: the widely used BLYP-D3 functional, with and without the EDS correction, and the meta-GGA SCAN functional. For all three DFT water models, the analysis of several structural and dynamical properties indicates that performing classical AIMD simulations at a higher temperature $(328 \mathrm{~K})$ introduces distinct and often opposite effects compared to performing quantum NQE (PIMD) simulations at 298 K. For MB-pol, the elevated temperature seems to have a similar softening effect as NQEs at the two-body level, but the threebody correlation, hydrogen-bond dynamics, and diffusivity are clearly affected by the higher temperature. These findings suggest that "mimicking" NQEs in water by performing classical MD simulations at an elevated temperature is problematic and, in some cases, quite misleading. Importantly, we consistently found that the over-structuring exhibited by these particular DFTbased AIMD models is further emphasized by NQEs. We note, however, that performing classical AIMD simulations with DFT models at an elevated temperature accidentally reduces the overstructuring issue in an ad hoc way. Based on our analyses, we conclude that the elevated temperature approach does not represent a physically correct way of effectively mimicking NQEs and requires further careful theoretical characterization and modification, including an examination of higher-order correlations and both molecular and collective dynamics, on a caseby-case basis, before being applied to other systems or to new water models (DFT-based or otherwise).

\section{Supporting Information}

The following files are available free of charge. 
Supporting figures showing the RDFs and tetrahedral order parameter computed on the imaginary time path centroid trajectories.

\section{Corresponding Author}

*gavoth@uchicago.edu

\section{Author Contributions}

The manuscript was conceived and written through contributions of all authors. All authors have given approval to the final version of the manuscript.

\section{ACKNOWLEDGMENTS}

This research was supported in part by the U.S. Department of Energy (DOE), Office of Basic Energy Sciences, Division of Chemical Sciences, Geosciences, and Biosciences under Award Numbers DE-SC0018648 (G.A.V.) and DE-SC0019490 (F.P.). The research was also supported in part by the National Institute of General Medical Sciences (NIGMS) of the U.S. National Institutes of Health (NIH) through grant R01 GM053148 (G.A.V). Computational resources were provided by the Research Computing Center (RCC) at the University of Chicago. We thank Dr. Paul Calio for analysis tools, Dr. Xinyou Ma for SCAN setup, and Won Hee (Harry) Ryu for discussions.

\section{REFERENCES}

1. Ceriotti, M.; W. Fang; P. G. Kusalik; R. H. McKenzie; A. Michaelides; M. A. Morales; T. E. Markland, Nuclear quantum effects in water and aqueous systems: Experiment, theory, and current challenges. Chem. Rev. 2016, 116, 7529-7550. 
2. Chen, B.; I. Ivanov; M. L. Klein; M. Parrinello, Hydrogen bonding in water. Phys. Rev. Lett. 2003, 91, 215503.

3. Habershon, S.; T. E. Markland; D. E. Manolopoulos, Competing quantum effects in the dynamics of a flexible water model. J. Chem. Phys. 2009, 131, 024501.

4. Morrone, J. A.; R. Car, Nuclear quantum effects in water. Phys. Rev. Lett. 2008, 101, 017801.

5. Marsalek, O.; T. E. Markland, Quantum dynamics and spectroscopy of ab initio liquid water: The interplay of nuclear and electronic quantum effects. J. Phys. Chem. Lett. 2017, 8, $1545-1551$.

6. $\quad$ Feynman, R. P.; A. R. Hibbs, Quantum mechanics and path integrals. McGraw-Hill: New York,, 1965; p xiv, 365 p.

7. Markland, T. E.; M. Ceriotti, Nuclear quantum effects enter the mainstream. Nat. Rev. Chem. 2018, 2, 1-14.

8. Markland, T. E.; D. E. Manolopoulos, An efficient ring polymer contraction scheme for imaginary time path integral simulations. J. Chem. Phys. 2008, 129, 024105.

9. Buxton, S. J.; S. Habershon, Accelerated path-integral simulations using ring-polymer interpolation. J. Chem. Phys. 2017, 147, 224107.

10. Ceriotti, M.; D. E. Manolopoulos, Efficient first-principles calculation of the quantum kinetic energy and momentum distribution of nuclei. Phys. Rev. Lett. 2012, 109, 100604.

11. Tuckerman, M. E., Ab initio molecular dynamics: basic concepts, current trends and novel applications. J. Phys.: Condens. Matter 2002, 14, R1297.

12. Pople, J. A.; P. M. Gill; B. G. Johnson, Kohn—Sham density-functional theory within a finite basis set. Chem. Phys. Lett. 1992, 199, 557-560.

13. Gillan, M. J.; D. Alfe; A. Michaelides, Perspective: How good is DFT for water? J. Chem. Phys. 2016, 144, 130901.

14. Hernández de la Peña, L.; P. G. Kusalik, Temperature dependence of quantum effects in liquid water. J. Am. Chem. Soc. 2005, 127, 5246-5251.

15. Paesani, F.; S. Iuchi; G. A. Voth, Quantum effects in liquid water from an ab initio-based polarizable force field. J. Chem. Phys. 2007, 127, 074506.

16. Yao, Y.; Y. Kanai, Temperature dependence of nuclear quantum effects on liquid water via artificial neural network model based on SCAN meta-GGA functional. J. Chem. Phys. 2020, $153,044114$. 
17. Yao, Y.; Y. Kanai, Nuclear Quantum Effect and Its Temperature Dependence in Liquid Water from Random Phase Approximation via Artificial Neural Network. J. Phys. Chem. Lett. 2021, 12, 6354-6362.

18. Ruiz Pestana, L.; O. Marsalek; T. E. Markland; T. Head-Gordon, The quest for accurate liquid water properties from first principles. J. Phys. Chem. Lett. 2018, 9, 5009-5016.

19. Sun, J.; A. Ruzsinszky; J. P. Perdew, Strongly constrained and appropriately normed semilocal density functional. Phys. Rev. Lett. 2015, 115, 036402.

20. Becke, A. D., Density-Functional Exchange-Energy Approximation with Correct Asymptotic-Behavior. Phys. Rev. A 1988, 38, 3098-3100.

21. Lee, C. T.; W. T. Yang; R. G. Parr, Development of the Colle-Salvetti CorrelationEnergy Formula into a Functional of the Electron-Density. Phys. Rev. B 1988, 37, 785-789.

22. Grimme, S.; J. Antony; S. Ehrlich; H. Krieg, A consistent and accurate ab initio parametrization of density functional dispersion correction (DFT-D) for the 94 elements H-Pu. $J$. Chem. Phys. 2010, 132, 154104.

23. Calio, P. B.; G. M. Hocky; G. A. Voth, Minimal Experimental Bias on the Hydrogen Bond Greatly Improves Ab Initio Molecular Dynamics Simulations of Water. J. Chem. Theory Comput. 2020, 16, 5675-5684.

24. Babin, V.; C. Leforestier; F. Paesani, Development of a "first principles" water potential with flexible monomers: Dimer potential energy surface, VRT spectrum, and second virial coefficient. J. Chem. Theory Comput. 2013, 9, 5395-5403.

25. Babin, V.; G. R. Medders; F. Paesani, Development of a "first principles" water potential with flexible monomers. II: Trimer potential energy surface, third virial coefficient, and small clusters. J. Chem. Theory Comput. 2014, 10, 1599-1607.

26. Medders, G. R.; V. Babin; F. Paesani, Development of a "first-principles" water potential with flexible monomers. III. Liquid phase properties. J. Chem. Theory Comput. 2014, 10, 29062910.

27. Reddy, S. K.; S. C. Straight; P. Bajaj; C. Huy Pham; M. Riera; D. R. Moberg; M. A. Morales; C. Knight; A. W. Götz; F. Paesani, On the accuracy of the MB-pol many-body potential for water: Interaction energies, vibrational frequencies, and classical thermodynamic and dynamical properties from clusters to liquid water and ice. J. Chem. Phys. 2016, 145, 194504.

28. Paesani, F., Getting the right answers for the right reasons: Toward predictive molecular simulations of water with many-body potential energy functions. Acc. Chem. Res. 2016, 49, 1844-1851.

29. Hartwigsen, C.; S. Gœdecker; J. Hutter, Relativistic separable dual-space Gaussian pseudopotentials from H to Rn. Phys. Rev. B 1998, 58, 3641. 
30. VandeVondele, J.; J. Hutter, An efficient orbital transformation method for electronic structure calculations. J. Chem. Phys. 2003, 118, 4365-4369.

31. Chen, M.; H.-Y. Ko; R. C. Remsing; M. F. C. Andrade; B. Santra; Z. Sun; A. Selloni; R. Car; M. L. Klein; J. P. Perdew, Ab initio theory and modeling of water. Proc. Natl. Acad. Sci. U. S. A. 2017, 114, 10846-10851.

32. Kuhne, T. D; M. Iannuzzi; M. Del Ben; V. V. Rybkin; P. Seewald; F. Stein; T. Laino; R. Z. Khaliullin; O. Schutt; F. Schiffmann; D. Golze; J. Wilhelm; S. Chulkov; M. H. BaniHashemian; V. Weber; U. Borstnik; M. Taillefumier; A. S. Jakobovits; A. Lazzaro; H. Pabst; T. Muller; R. Schade; M. Guidon; S. Andermatt; N. Holmberg; G. K. Schenter; A. Hehn; A. Bussy; F. Belleflamme; G. Tabacchi; A. Gloss; M. Lass; I. Bethune; C. J. Mundy; C. Plessl; M. Watkins; J. VandeVondele; M. Krack; J. Hutter, CP2K: An electronic structure and molecular dynamics software package - Quickstep: Efficient and accurate electronic structure calculations. J. Chem. Phys. 2020, 152, 194103.

33. VandeVondele, J.; M. Krack; F. Mohamed; M. Parrinello; T. Chassaing; J. Hutter, QUICKSTEP: Fast and accurate density functional calculations using a mixed Gaussian and plane waves approach. Comput. Phys. Commun. 2005, 167, 103-128.

34. Tribello, G. A.; M. Bonomi; D. Branduardi; C. Camilloni; G. Bussi, PLUMED 2: New feathers for an old bird. Comput. Phys. Commun. 2014, 185, 604-613.

35. Lehtola, S.; C. Steigemann; M. J. T. Oliveira; M. A. L. Marques, Recent developments in LIBXC - A comprehensive library of functionals for density functional theory. Softwarex $\mathbf{2 0 1 8 ,}$ 7, 1-5.

36. Wang, H.; L. Zhang; J. Han; E. Weinan, DeePMD-kit: A deep learning package for many-body potential energy representation and molecular dynamics. Comput. Phys. Commun. 2018, 228, 178-184.

37. Zhang, L.; J. Han; H. Wang; W. Saidi; R. Car; W. E In End-to-end Symmetry Preserving Inter-atomic Potential Energy Model for Finite and Extended Systems, Advances in Neural Information Processing Systems, Bengio, S.; Wallach, H.; Larochelle, H.; Grauman, K.; CesaBianchi, N.; Garnett, R., Eds. Curran Associates, Inc.: 2018.

38. Plimpton, S., Fast Parallel Algorithms for Short-Range Molecular-Dynamics. J. Comput. Phys. 1995, 117, 1-19.

39. Kapil, V.; M. Rossi; O. Marsalek; R. Petraglia; Y. Litman; T. Spura; B. Cheng; A. Cuzzocrea; R. H. Meißner; D. M. Wilkins, i-PI 2.0: A universal force engine for advanced molecular simulations. Comput. Phys. Commun. 2019, 236, 214-223.

40. Rossi, M.; M. Ceriotti; D. E. Manolopoulos, How to remove the spurious resonances from ring polymer molecular dynamics. J. Chem. Phys. 2014, 140, 234116. 
41. Reddy, S. K.; D. R. Moberg; S. C. Straight; F. Paesani, Temperature-dependent vibrational spectra and structure of liquid water from classical and quantum simulations with the MB-pol potential energy function. J. Chem. Phys. 2017, 147, 244504.

42. Skinner, L. B.; C. Huang; D. Schlesinger; L. G. Pettersson; A. Nilsson; C. J. Benmore, Benchmark oxygen-oxygen pair-distribution function of ambient water from x-ray diffraction measurements with a wide Q-range. J. Chem. Phys. 2013, 138, 074506.

43. Soper, A., The radial distribution functions of water and ice from 220 to $673 \mathrm{~K}$ and at pressures up to $400 \mathrm{MPa}$. Chem. Phys. 2000, 258, 121-137.

44. Soper, A.; C. Benmore, Quantum differences between heavy and light water. Phys. Rev. Lett. 2008, 101, 065502 .

45. Chau, P.-L.; A. Hardwick, A new order parameter for tetrahedral configurations. Mol. Phys. 1998, 93, 511-518.

46. Giberti, F.; A. A. Hassanali; M. Ceriotti; M. Parrinello, The role of quantum effects on structural and electronic fluctuations in neat and charged water. J. Phys. Chem. B 2014, 118, 13226-13235.

47. Cassone, G., Nuclear quantum effects largely influence molecular dissociation and proton transfer in liquid water under an electric field. J. Phys. Chem. Lett. 2020, 11, 8983-8988.

48. Luzar, A.; D. Chandler, Hydrogen-bond kinetics in liquid water. Nature 1996, 379, 5557.

49. Yeh, I.-C.; G. Hummer, System-size dependence of diffusion coefficients and viscosities from molecular dynamics simulations with periodic boundary conditions. J. Phys. Chem. B 2004, 108, 15873-15879.

50. Holmes, M.; N. Parker; M. Povey In Temperature dependence of bulk viscosity in water using acoustic spectroscopy, Journal of Physics: Conference Series, IOP Publishing: 2011; p 012011.

51. Holz, M.; S. R. Heil; A. Sacco, Temperature-dependent self-diffusion coefficients of water and six selected molecular liquids for calibration in accurate 1H NMR PFG measurements. Phys. Chem. Chem. Phys. 2000, 2, 4740-4742. 


\section{TOC Figure}
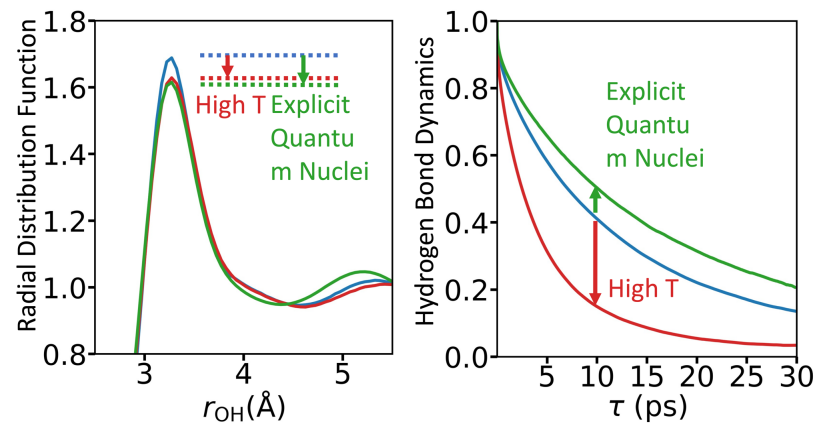